careful -[t-i]. (4) idet Ir'ina goes Irina fast $-[\mathrm{t}-\mathrm{i}]$; careful $-[\mathrm{t}-\mathrm{i}]$. Formants of [i] versus [-i] were measured to examine coarticulation, as evidenced by a barred $\mathrm{i}$ in the phonetic realization of /i/. Results showed that (A) stressed V1 of the final word has no coarticulatory effects in careful speech, whereas (B) stressed V2 adhered to the rule.

2aSC14. Effects of syllable structure and syllable boundary on segment duration in Seoul Korean. Byung-jin Lim (Dept. of Linguist., Indiana Univ., Bloomington, IN 47405, bylim@indiana.edu)

The present study reports results of an investigation into Korean durational patterns with respect to syllable structure and syllable boundary. The main questions raised in this study were (1) whether different preceding syllable structures contribute to the durational patterns of the following segmental durations: closure duration, voice onset timing (VOT), and vowel duration; and, if so, (2) how they are realized, especially over syllable boundaries determined by Korean orthography. Thirty-six twosyllable nonsense words varying in syllable structure were analyzed. For the durational pattern of internal elements of syllables over syllable boundary, the initial consonants of the second syllable were differentiated into lax, tensed, and aspirated stops. Results indicate that initial consonant duration showed no significant difference regardless of syllable structure. Initial vowel duration, however, reflected temporal compensation depending on the syllable structure. In addition, the effect of preceding syllable structure was reflected only on the closure duration, not on the VOT nor on the vowel durations of following syllables. These findings imply that temporal compensation tends to occur across segments over syllable boundaries, suggesting the importance of syllable boundary in durational patterns in Seoul Korean. The relevance of orthography to syllable boundary is also discussed.

2aSC15. The effect of neutral tone on the $f_{0}$ peak alignment in Standard Chinese. Zhiqiang Li (Dept. of Linguist., MIT, 77 Massachusetts Ave., Cambridge, MA 02139, zqli@mit.edu)

The $f_{0}$ peaks sometimes are realized after the right-hand boundary of their host syllables when followed by syllables that carry one of the four tones in Standard Chinese. Further, tone $1(H)$ and tone $2(R)$ exhibit different peak alignment patterns. This study investigates the effect of neutral tone on the alignment of $f_{0}$ peaks with their host syllables in tone 1 and tone 2 at normal speaking rate. When a syllable is unstressed, it does not carry any of the four tones, but is said to be in the neutral tone. The greatest amount of peak delay was found on tone 2 when it was followed by one or two neutral tone syllables. The $f_{0}$ peaks were aligned pretty close to the $C-V$ boundary of the following syllable, and in many cases even across it. The peak on tone 1 was delayed the most in the context of two neutral tone syllables, always being realized after the right-hand boundary of its host syllable, and the least before a full tone syllable. The different alignment patterns are attributable to the inherent tonal properties of tone 1 and tone 2, and the phonological analysis of neutral tone.

2aSC16. Intrinsic versus interaction effects on $\boldsymbol{F 0}$. Mi-Ryoung Kim (Program in Linguist., Univ. of Michigan, 1076 Frieze Bldg., Ann Arbor, MI 48109-1285)

This study investigates the nature and magnitude of the effect of segments, initial and final consonants, vowel quality, and vowel length on $F 0$ contour in English and Korean. The two languages were compared with a special reference of the effect of initial consonants on $F 0$. F0 was measured at three temporal locations of each syllable rime onset, midpoint, and offset. For Korean, of the four segment types, initial consonants had the greatest effect on $F 0$ contour in both spectral and temporal magnitude, indicating that consonant types interacted with tone. The English data showed that the segmental influence reflected intrinsic effects on $F 0$. In particular, the effects of initial consonants on $F 0$ contours in English were significantly smaller in spectral and temporal extent than those of Korean. This study concluded that there are cross-linguistic differences between English and Korean concerning the effect of initial consonants on $F 0$ contour (which I call "intrinsic" versus "interaction"). The consonanttone interactions in Korean are viewed as consistent with tonogenesis: voiceless (aspirated and tense) consonants correlate lead $\mathrm{H}$ and phonologically voiced (lax, sonorants, and zero) consonants lead LH. This is the first study that accounts for the Korean interaction phenomena by a theory of tonogenesis.

2aSC17. Recoverability constraints on gestural overlap in Georgian stop sequences. Ioana Chitoran (Linguist. and Cognit. Sci., Dartmouth College, 6087 Dartmouth Hall, Hanover, NH 03755, ioana.chitoran@dartmouth.edu), Dani Byrd (Univ. of Southern California, Los Angeles, CA 90025, dbyrd@usc.edu), and Louis Goldstein (Yale Univ. and Haskins Labs., New Haven, CT 06510)

Recent investigations of the temporal organization of articulatory gestures have found that consonants in a cluster exhibit less temporal overlap when in word-onset position than when the cluster occurs word-finally or spanning a word boundary. A possible reason for this is that substantial overlap of obstruent gestures in utterance-initial position may threaten their perceptual recoverability. Recoverability considerations may also account for results showing that a front-to-back order of place of articulation in stop-stop sequences (labial-alveolar, alveolar-velar, labial-velar) allows more overlap than the opposite order. Presumably, the recoverability of $\mathrm{C} 1$ is hindered if the release of $\mathrm{C} 1$ has no acoustic manifestation due to the presence of a more anterior $\mathrm{C} 2$ being coproduced in time, hiding the C1 release. Data demonstrating both these constraints on gestural patterning have previously been drawn only from English, limiting the type and position of consonant sequences. The Georgian language offers a rich inventory of consonant sequences including stop-stop sequences in initial, medial, and cross-word-boundary positions. This work is an articulator movement tracking study of two Georgian speakers producing such sequences. The results provide evidence that the sequence's word position and the component consonants' constriction locations both constrain the patterns of gestural overlap produced. [Work supported by NIH.]

2aSC18. On the noncategorical perception of Cantonese level tones. Alexander L. Francis, Brenda K. C. Ng, and Valter Ciocca (Dept. of Speech and Hearing Sci., Univ. of Hong Kong, Hong Kong)

Previous research provides conflicting evidence about the categorical nature of lexical tone perception. Chan et al. [J. Acoust. Soc. Am. 58, 119 (1975)] found that an $f_{0}$ continuum between a Mandarin level tone (high level) and a contour tone (high rising) was perceived categorically. In contrast, Abramson [J. Acoust. Soc. Am. 33, 842(A) (1961)] found that a Thai level tone continuum ranging in $f_{0}$ from low level to high level was not perceived categorically. The present study examined whether level lexical tones are perceived categorically in Cantonese. Native Cantonese speakers were asked to identify and discriminate stimuli consisting of Klatt-synthesized Cantonese syllables ranging in $f_{0}$ from low level to high level in nine equal mel steps. For identification, syllables were presented medially in (synthesized) sentential context to facilitate talker-dependent pitch processing [P. Wong and R. Diehl, J. Acoust. Soc. Am. 104, 1834 (1998)]. For discrimination, adjacent steps were presented in isolated pairs with $250 \mathrm{msec}$ ISI. Although the identification function showed boundaries typical of categorically perceived continua, discrimination was poor 
across the continuum. These results suggest that Cantonese level tones are not perceived categorically. Results and implications for theories of tone perception will be discussed.

2aSC19. A study of vowel length in Thai. Rungpat Roengpitya (Univ. of California at Berkeley, 1203 Dwinelle Hall, Berkeley, CA 94720)

In Thai, vowel length is contrastive (e.g., [cip] "to sip"-[ciip] "to pleat"). The main cue to distinguish short-long vowels in Thai is vowel duration [Abramson, 1962]. However, in some literature, there are other cues for vowel length in Thai, such as final nasal duration. This paper was aimed to see how important vowel duration and final nasal duration are as perceptual cues for Thai listeners. A perceptual experiment was conducted. Eighteen (near-) minimal pairs of Thai words were used, consisting of initial voiceless unaspirated stop [p], nine pairs of Thai vowels, a final voiceless velar stop [k] or an alveolar final nasal [n]. Short vowels were lengthened and long vowels were shortened at 10-30-ms steps, using LPC analysis and resynthesis. The same procedure was done for short and long final nasals at 10 -ms steps. All 1148 tokens were put into a Thai frame sentence and randomized. Forty native Thai listeners listened to these tokens and judged whether each token had a short or a long vowel. The results are that vowel duration is the main perceptual cue to distinguish short and long vowels in Thai.

\title{
Session 2aSP
}

\section{Signal Processing in Acoustics, Biomedical Ultrasound/Bioresponse to Vibration, Underwater Acoustics, and Acoustical Oceanography: Acoustical Imaging and Tomography}

\author{
David H. Chambers, Chair \\ Lawrence Livermore National Laboratory, P.O. Box 5508, Livermore, California 94551-5508
}

Chair's Introduction-8:25

Invited Papers

8:30

2aSP1. Quantitative three-dimensional imaging with diffracting wavefields. Anthony J. Devaney (Dept. of Elec. and Computer Eng., Northeastern Univ., Boston, MA 02115)

The general theory of quantitative imaging of three-dimensional, semi-transparent (weakly scattering) objects using scalar waves is presented. The theory is developed for the case of transmission type experiments appropriate to ultrasound diffraction tomography but is applicable with minor modifications to reflection geometries appropriate to pulse echo imaging. The imaging problem is formulated from first principles in terms of the three-dimensional scalar wave equation and is shown to reduce ultimately to an inverse scattering problem. By use of certain linearizing approximations the inverse problem is shown to reduce to a conventional (coherent) imaging problem having a well-defined point spread function (PSF) that can be computed in terms of the experimental parameters and imaging geometry. It is shown that this formulation allows quantitative three-dimensional imaging to be treated completely analogously to coherent optical imaging and, in particular, leads to a characterization of image quality in terms of the PSF and its spatial Fourier transform, the coherent transfer function (CTF). Inherent limitations of three-dimensional imaging are discussed based on the computed PSF for certain canonical geometries. These limitations are shown to be partially overcome by using suites of scattering experiments and/or beam scanning techniques such as focus-on-transmit and focus-on-receive. The talk includes a discussion of the validity of the weak scattering approximations that underlie the imaging model as well as a discussion of the use of the wave aberration function for characterizing image quality. The talk is illustrated with simulated and experimental results.

\section{9:00}

2aSP2. Computing resolution in acoustic tomography. James G. Berryman (LLNL, P.O. Box 808 L-200, Livermore, CA 94551-9900)

The resolution of images obtained using acoustic tomography is important both after the fact, as a means of evaluating the usefulness of the final image, and before the fact, when the measurement system of sources and receivers is being designed according to some preset criteria specified by the users. Resolution computations can actually be significantly more difficult and expensive than computing the image itself. Our purpose then is to show how resolution can be computed economically in almost all cases, and to provide a means of comparing the resolution characteristics of many of the common approximate inverse methods. Means of obtaining the effective resolution operator for such standard iterative inversion procedures as conjugate gradients, Lanczos, and LSQR are provided while avoiding the more common approach of producing a singular-value decomposition (SVD) of the operator being inverted. The methods discussed produce very easy-to-understand results for purposes of analysis, but require reorthogonalization of the Krylov vectors/operators produced by realistic iterative procedures in finite precision. Although the need for reorthogonalization increases the expense of the procedure somewhat, it still produces the desired results with much greater efficiency than could be obtained by computing an SVD of the full operator. 